\title{
The Steady-State Comparative Bioavailability of Intramuscular Risperidone ISM and Oral Risperidone: An Open-Label, One-Sequence Study
}

\author{
David P Walling (D) \\ Howard A Hassman ${ }^{2}$ \\ Lourdes Anta (D) ${ }^{3}$ \\ Lourdes Ochoa (iD) 4 \\ Ignacio Ayani $\mathbb{D}^{3}$ \\ Javier Martínez $\mathbb{D}^{3}$ \\ Ibon Gutierro (iD ${ }^{4}$ \\ 'Collaborative Neuroscience Network, \\ LLC Garden Grove, Garden Grove, CA, \\ USA; ${ }^{2}$ Hassman Research Institute, \\ Berlin, NJ, USA; ${ }^{3}$ Medical Department, \\ Laboratorios Farmacéuticos ROVI, S.A., \\ Madrid, Spain; ${ }^{4}$ R\&D Department, \\ Laboratorios Farmacéuticos ROVI, S.A., \\ Madrid, Spain
}

Correspondence: Javier Martínez Laboratorios Farmacéuticos ROVI, S.A., Calle Alfonso Gómez, 45A, Madrid, 28037, Spain

Tel + 34913756336

$\mathrm{Fax}+34913047881$

Email jmartinez@rovi.es
Introduction: This open-label, one-sequence study evaluated the steady-state comparative bioavailability of risperidone in situ microimplants $\left(\mathrm{ISM}^{\circledR}\right)$ and oral risperidone in patients stabilized on oral risperidone treatment.

Methods: Repeat oral administration of once daily $4 \mathrm{mg}$ risperidone for 7 days was followed by 4 monthly (once every four weeks) intramuscular (IM) doses of risperidone ISM $100 \mathrm{mg}$. Mean steady-state concentration versus time profiles for risperidone, 9-OH risperidone, and risperidone active moiety was characterized.

Results: A total of 104 subjects were enrolled, 81 were included in the safety population and 58 completed the study. Intersubject variability for the steady-state concentrations versus time profiles for risperidone active moiety presented a greater variability range for oral risperidone versus risperidone ISM (\% coefficient of variation [CV] range: $40-65 \%$ and $38-52 \%$, respectively). Minimum plasma concentration at steady-state $\left(\mathrm{C}_{\min }, \mathrm{ss}\right)$ and fluctuation in plasma concentrations (Fluc) of risperidone active moiety after risperidone ISM administration met bioequivalence criteria compared to the reference oral risperidone (geometric mean ratio [GMR] $=1.09$ and 0.96, respectively; both $90 \%$ CIs were within 0.80-1.25). Area under the curve during the dosing interval $\left(\mathrm{AUC}_{\mathrm{tau}}\right)$, maximum plasma concentration at steady-state $\left(\mathrm{C}_{\mathrm{max}}, \mathrm{ss}\right)$ and average plasma concentration $\left(\mathrm{C}_{\mathrm{ave}}\right)$ were only slightly higher $(\mathrm{GMR}[90 \% \mathrm{CI}]=1.25[1.16$ 1.34], 1.17 [1.08-1.27], and 1.25 [1.16-1.34], respectively). Overall, once daily oral risperidone $4 \mathrm{mg}$ and once monthly IM risperidone ISM $100 \mathrm{mg}$ were generally safe and well tolerated in the participating subjects with schizophrenia previously stabilized with oral risperidone.

Conclusion: The rapid release of risperidone ISM allows the achievement of the desired levels similar to those observed at the steady-state after oral risperidone treatment. Therefore, direct switch after 24 hours from the last oral risperidone dose to risperidone ISM treatment can be done in schizophrenia patients with no time lag, maintaining steady-state levels of the active moiety throughout treatment and without the need for oral risperidone supplementation or loading doses. Keywords: bioequivalence, comparative bioavailability, long-acting injectables, LAIs, pharmacokinetic, risperidone, schizophrenia

\section{Introduction}

One of the most important issues in the treatment of schizophrenia is suboptimal adherence to oral antipsychotic medications, which has been associated with risk of relapse and disease progression. ${ }^{1}$ Oral risperidone is a commonly used medication for 
the treatment of schizophrenia and related psychotic disorders, and long-acting injectable (LAI) antipsychotics have been used in patients with frequent relapses. The latter is an important treatment option to improve adherence and efficacy. ${ }^{2,3}$ Risperidone In Situ Microimplants $\left(\right.$ ISM $^{\circledR}$ ) is a new intramuscular (IM) LAI formulation providing plasma levels in the therapeutic range within the first hours after administration which are maintained for up to one month without the need for oral risperidone supplementation or loading doses. ${ }^{4,5}$ The ISM technology used in this formulation is based on a solid and stable polymeric matrix system that contains risperidone. The product is reconstituted to an injectable suspension that precipitates in situ (inside the body) after IM injection, resulting in the formation of a matrix, by solvent diffusion to body fluids. This matrix biodegrades slowly, providing a sustained and controlled release of drug for up to 1 month. ${ }^{6}$ The ISM technology enables the extended delivery of compounds with the following advantages: less variability, enhanced stability, rapid reconstitution, and straightforward injection, making it uncomplicated for the patient and provider to follow the prescribed treatment. ${ }^{4}$

The aim of this study was to evaluate the steady-state comparative bioavailability, safety, and tolerability of risperidone ISM and oral risperidone, as well as provide evidence that the direct switch from oral risperidone to Risperidone ISM is appropriate.

\section{Methods}

\section{Study Design}

This was an open-label, one-sequence study conducted between 07/2018 and 03/2019 at two sites in the United States (Hassman Research Institute and Collaborative Neuroscience Network), in accordance with the Declaration of Helsinki, and Good Clinical Practice principles outlined in the International Conference on Harmonization. The protocol, amendments, and informed consent were approved by the institutional review boards (IRB) of both sites, and written informed consent was obtained from all subjects before study participation. The initial IRB approval was obtained on June 14, 2018, from Aspire IRB (for Hassman Research Institute) and on May 14, 2018, from Alpha IRB (for Collaborative Neuroscience Network). This study was registered at ClinicalTrials.gov (NCT 03527186).

The study consisted of a screening visit, 1 treatment period with inpatient and outpatient visits, and a follow-up visit. Subjects who were already receiving oral risperidone treatment $(4 \mathrm{mg}$ ) continued the oral regimen for 1 week (from Day 1 to Day 7) to achieve steady-state concentrations of risperidone. Following the oral risperidone treatment, a single IM dose of $100 \mathrm{mg}$ Risperidone ISM was administered on Day 8 into the gluteal muscle. A total of 4 monthly (once every four weeks) IM doses were injected.

\section{Patients}

Eligible subjects were $18-65$ years old with a current diagnosis of schizophrenia according to the Diagnostic and Statistical Manual of Mental Disorders, Fifth Edition (DSM-5), criteria. All subjects were medically stable during the preceding month with a body mass index (BMI) of 17$35 \mathrm{~kg} / \mathrm{m}^{2}$ and were receiving oral risperidone $4 \mathrm{mg}$ daily as maintenance therapy for at least the last 4 weeks prior to screening and on $4 \mathrm{mg}$ oral risperidone once daily for at least one-week prior to baseline (Day 1). Subjects were outpatients free from significant symptom exacerbation and had not been hospitalized for worsening of schizophrenia during the preceding 3 months and had a Clinical Global Impression Severity (CGI-S) score of $\leq 4$ (moderately ill). ${ }^{7}$

Reasons for exclusion included the presence of an uncontrolled, unstable or clinically significant medical condition, examination finding or abnormality on laboratory test that could interfere with the conduct of the study or compromise the well-being of the subject or evidence of a significant electrocardiogram (ECG) abnormality. Subjects were also excluded if an imminent risk of selfharm or causing harm to others, based on clinical interview and responses provided on the Columbia-Suicide Severity Rating Scale (C-SSRS), ${ }^{8}$ was observed; if subjects had reported suicidal ideation of Type 4 or 5 in the previous 2 months, or suicidal behavior in the previous 6 months; use of depot antipsychotics within the last 6-9 months or if they had been taking more than one antidepressant or if taking just one, a change in dose within the last 4 weeks prior to screening. Women who were pregnant or breastfeeding were also excluded.

\section{Treatment}

No randomization was used in this study.

Risperidone ISM 100 mg (Laboratorios Farmacéuticos ROVI, S.A., Madrid, Spain) was supplied in a kit of two syringes, one containing risperidone plus poly lactic-coglycolic acid (PLGA), and the other containing dimethyl sulfoxide (DMSO), the solvent required for reconstitution. 
Tablets of oral risperidone $4 \mathrm{mg}$ (Risperdal; Janssen Ortho LLC, Gurabo, Puerto Rico, US) were also supplied.

\section{Assessments of Bioavailability}

Venous blood samples, to determine the plasma concentrations of risperidone, its active metabolite $9-\mathrm{OH}$ risperidone and the active moiety (risperidone plus 9-OH-risperidone), were collected on Days 1-6 pre-dose; Day 7: pre-dose and at 1, 2, 3, 4, 6, 8, and 12 hours post-dose; Day 8 pre-dose and 12 hours post-dose; Days 10, 15, 22, 29, 36 (pre-dose) , and 64 (pre-dose); Day 92 pre-dose and at 2, 8, and 12 hours after dosing; and Days 93, 94, 97, 99, 102, 106, 110, 113,117 , and 120 .

The pharmacokinetic (PK) parameters were determined for risperidone, 9-OH risperidone, and the active moiety. As risperidone and 9-OH-risperidone have similar pharmacological activity; the combined PK is merged into the active moiety.

The following plasma PK parameters were determined at steady-state (situation where the overall intake of a drug is in fairly dynamic equilibrium with its elimination), after the seventh dose of oral risperidone (Day 7), and after the fourth dose of risperidone ISM (Day 92): area under the plasma concentration versus time curve during the dosing interval $\left(\mathrm{AUC}_{\mathrm{tau})}\right.$, average plasma concentration $\left(\mathrm{C}_{\mathrm{ave}}\right)$, minimum plasma concentration at steady-state $\left(\mathrm{C}_{\min } \mathrm{ss}\right)$, maximum plasma concentration at steady-state $\left(\mathrm{C}_{\max s s}\right)$, time to the maximum plasma concentration at steady-state ( $\mathrm{T}_{\max \mathrm{ss}}$ ), and percent fluctuation.

The following PK parameters were determined following the first dose of risperidone ISM (Day 8): $\mathrm{C}_{\mathrm{ave}}$, minimum plasma concentration $\left(\mathrm{C}_{\min }\right)$, maximum plasma concentration $\left(\mathrm{C}_{\max }\right)$, time to maximum plasma concentration $\left(\mathrm{T}_{\max }\right)$, and percentage peak to trough fluctuation over a dosing interval (Fluc).

The primary $\mathrm{PK}$ endpoint was the steady-state $\mathrm{AUC}_{\mathrm{tau}}$ for the active moiety. The steady-state $\mathrm{AUC}_{\text {tau }}$ for oral risperidone was calculated for the 24-hour dosing interval following dosing on Day 7 and prior to the first administration of risperidone ISM on Day 8.

The steady-state $\mathrm{AUC}_{\text {tau }}$ for risperidone ISM was calculated for the 28-day period following administration of the fourth dose of risperidone ISM interval on Day 92 up to the final blood sample obtained on Day 120.

The secondary PK endpoints included steady-state $\mathrm{AUC}_{\text {tau }}$ for risperidone and 9-OH risperidone separately, steady-state $\mathrm{C}_{\mathrm{ave}}$ for the active moiety, risperidone, and 9-OH risperidone separately, steady-state trough level $\left(\mathrm{C}_{\min } \mathrm{ss}\right)$ and peak level $\left(\mathrm{C}_{\max s s}\right)$ for the active moiety, risperidone, and 9-OH risperidone separately, steady-state percent fluctuation for the active moiety, risperidone, and 9-OH risperidone separately, among others.

\section{Assessments of Safety}

Safety and tolerability were assessed by monitoring adverse events (AEs), laboratory test results, vital signs, ECG results, physical examination and psychometric scales to evaluate severity of illness (CGI-S), ${ }^{7}$ extrapyramidal symptoms (Abnormal Involuntary Movement Scale, AIMS, ${ }^{9}$ Barnes Akathisia Rating Scale, BARS, ${ }^{10}$ and Simpson Angus Scale, SAS), ${ }^{11}$ suicidality $(\mathrm{C}-\mathrm{SSRS})^{8}$ as well as injection site reactions (redness, swelling and induration) and injection site pain, using Visual Analog Scale (VAS).

Treatment-emergent adverse events (TEAEs) were defined as AEs that occurred or worsened after the first dose of study drug. The TEAEs were differentiated if they occurred during oral risperidone administration or after risperidone ISM injection. The incidence of treatmentrelated TEAEs, serious TEAEs and TEAEs leading to discontinuation from the study are presented.

\section{Assessments of Pharmacogenomics}

A blood sample was collected for evaluation of genotypes for cytochrome P450 (CYP) enzymes (CYP2D6 genotype) and/or genes that were potentially related to efficacy response and/or adverse effects. The sample could be obtained immediately after enrollment into the study. The samples were tested for subjects who signed a separate consent form for correlation with PK and/or efficacy results.

\section{Statistical Analysis}

It was calculated that approximately 65 (no screening failure) subjects were needed to be enrolled, with the intent to complete tests on 41 subjects. Sixty-five subjects were estimated for enrollment based on 41 completers assuming an approximate drop-out rate of $37 \%$.

The power to produce a $90 \%$ confidence interval for the geometric mean ratio, which lies within $80 \%$ to $125 \%$ for $\mathrm{AUC}_{\text {tau }}$ from 41 completed subjects, was calculated for different scenarios of the true intra-subject $\% \mathrm{CV}$ and true geometric mean ratio.

All analyses were performed using SAS $^{\circledR}$ version 9.4 (SAS Institute, Inc, Cary, North Carolina).

The enrolled population included all subjects enrolled in the study, who signed the informed consent form. The safety population included all subjects who received at 
least 1 dose of study drug. The PK population included all subjects in the safety population who completed the study and had no major protocol violations that would exclude them from analysis. The PGx population included all subjects in the safety population who had available genotyping data from screening.

The PK parameters for risperidone, 9-OH risperidone and the active moiety were derived by applying a noncompartmental approach using Phoenix ${ }^{\circledR}$ WinNonLin ${ }^{\circledR} 8.0$ (Pharsight Corporation, St Louis, Missouri) or $\mathrm{SAS}^{\circledR}$ Version 9.4.

Descriptive statistics (arithmetic mean, standard deviation [SD], coefficient of variation [CV], median, minimum, and maximum) were calculated by time point and treatment.

PK parameters were listed by individual and summarized by treatment and day by using descriptive statistics (arithmetic mean, $\mathrm{SD}, \mathrm{CV}$, geometric mean, geometric $\mathrm{CV}$, median, minimum, and maximum). Summary statistics for categorical parameters, such as $\mathrm{T}_{\max }$, included median, minimum, and maximum only. Steady-state PK parameters were also summarized by treatment and CYP2D6 phenotype.

To determine the relative comparability of steady-state plasma exposure between $4 \mathrm{mg}$ oral risperidone and $100 \mathrm{mg}$ Risperidone ISM, natural $\log$ transformation of the adjusted $\mathrm{AUC}_{\text {tau }}, \mathrm{C}_{\max \text { ss }}, \mathrm{C}_{\text {min ss }}, \mathrm{C}_{\mathrm{ave}}$, and Fluc for risperidone, 9-OH risperidone, and the active moiety was performed and values were compared between treatments by using an analysis of variance with treatment as a fixed effect and subject as a random effect. The corresponding means, mean differences, and $90 \%$ confidence interval (CI) for the mean difference (based on the standard error and t-distribution) were back transformed (exponentiated) to derive the geometric means, geometric mean ratio (GMR), and 90\% CI for the GMR for each parameter. Acceptance criteria for the $90 \%$ CI for the GMR of $0.80-1.25$ was applied.

To determine differences in $T_{\max }$ ss between oral risperidone and risperidone ISM, the Wilcoxon signed rank test was performed.

To assess whether steady-state was achieved for each treatment, aggregate assessment of trough concentrations $\left(\mathrm{C}_{\text {min }}\right.$ ss $)$ by repeated-measures analysis of variance (Helmert Contrast Transformation) with dose as a fixed effect and measurement within each subject as repeated measures was conducted. The number of doses necessary to achieve steady-state for Risperidone ISM and oral risperidone were determined. ${ }^{12}$

In addition, steady-state was assessed by using a mixed model ${ }^{13}$ with repeated measures performed on $\mathrm{C}_{\text {min ss }}$ for three analytes, with study day as a covariate and subject as a random effect. A random slope effect for study day was also included. The linear effect of study day on $\mathrm{C}_{\min }$ ss over time was used to assess the assumption of steadystate.

\section{Bioanalytical Method}

An HPLC/MS/MS method for the determination of risperidone and 9-OH-risperidone in human K2-EDTA plasma was validated according to the FDA Guidance for Industry: Bioanalytical Method Validation over an analytical range of 100 to $50,000 \mathrm{pg} / \mathrm{mL}$ for both analytes. The analytical methodology was based on an automated liquidliquid extraction employing $0.2 \mathrm{~mL}$ of plasma sample and using d4-risperidone and $\mathrm{d} 4-9-\mathrm{OH}$-risperidone as internal standard. The in-study method performance was evaluated. The within-run and between-run accuracy ranged from 0.91 to $0.57 \%$ for risperidone and -0.77 to $0.57 \%$ for 9-OH-risperidone respectively. The precision of quality control samples ranged from 6.48 to $8.75 \%$ for risperidone and 4.43 to $6.65 \%$ for $9-\mathrm{OH}$-risperidone respectively.

\section{Results}

From a total of 104 subjects assessed for eligibility, 81 received at least 1 dose of study drug. Of those, 58 completed the study and 23 discontinued (Figure 1).

During oral risperidone treatment, 73 subjects $(90.1 \%)$ received all 7 doses of the study drug. And during Risperidone ISM treatment, 58 subjects $(79.5 \%)$ received all 4 doses of the study drug.

The safety, PK and PGx populations included 81, 58, and 39 subjects, respectively.

Subject demographic and baseline characteristics are summarized in Table 1. Most subjects were male and black or African American with a mean age of 49.2 years and a mean BMI of $27.96 \mathrm{~kg} / \mathrm{m}^{2}$.

Eighteen (46.2\%) and 17 (43.6\%) subjects were extensive or intermediate metabolizers, while 4 (10.3\%) subjects were ultra-metabolizers, and none were poor metabolizers.

\section{Pharmacokinetic Evaluation}

Ten subjects were excluded from the PK statistical analysis because steady-state was not achieved for them on oral 
Screening Failures $(n=23)$

- Laboratory results out of range: $6(26.1 \%)$

- Medical history exclusión: $5(21.7 \%)$

- Withdrawal of consent: $2(8.7 \%)$

- Other: $10(43.5 \%)$

Drop-out $(n=8)$

- Withdrawal of consent: $7(8.6 \%)$

- AEs: $1(1.2 \%)$
Potentially elegible participants $(n=104)$

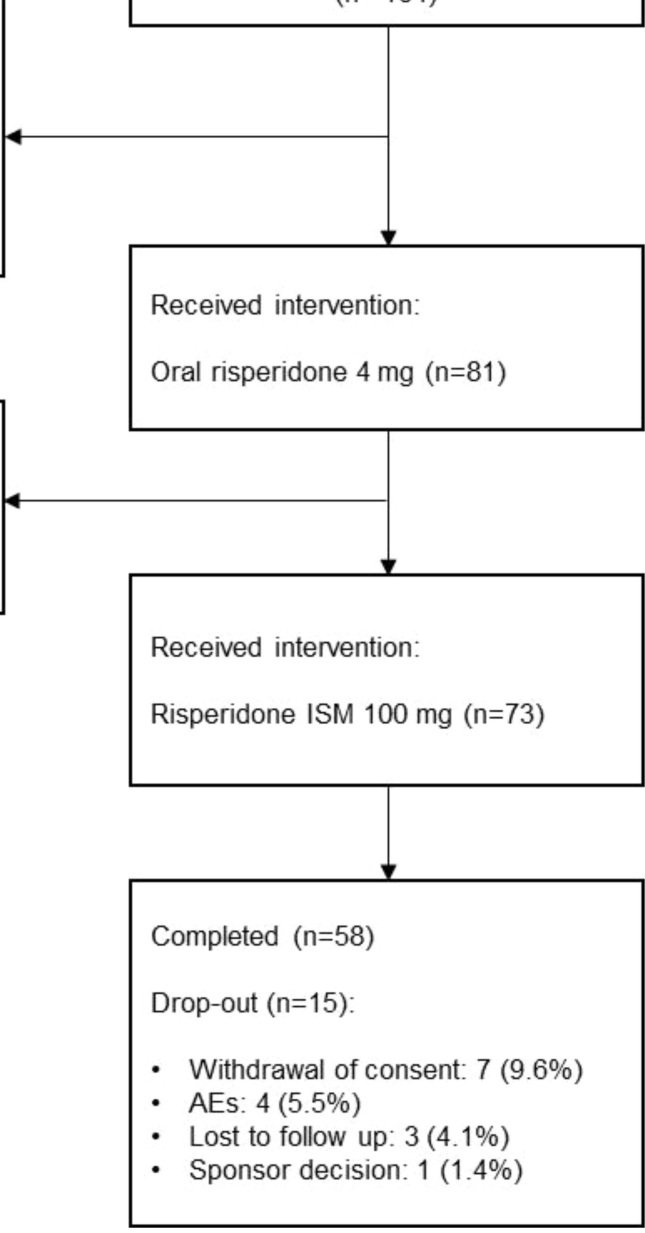

Figure I Subject disposition.

risperidone treatment. These subjects were also excluded from the analysis of Risperidone ISM treatment to maintain a balanced sample size.

\section{Plasma Concentrations}

Following repeated oral administration of once daily $4 \mathrm{mg}$ risperidone for 7 days, mean steady-state concentration versus time profiles for risperidone active moiety were characterized by a steady absorption phase, reaching peak values with a median $\mathrm{T}_{\max }$ ss of 2 hours, followed by a monophasic decrease in concentrations to 24 hours post-dose (Figure 2; Supplementary Figure 1).

The first IM dose of risperidone ISM $100 \mathrm{mg}$ was administered 24 hours after the last oral dose, without any washout period. From the first measurement after the first injection (12 hours), mean active moiety plasma concentrations achieved similar levels to those observed on oral treatment in steady-state and were maintained above the therapeutic threshold $(>7.5 \mathrm{ng} / \mathrm{mL})^{14}$ throughout the dosing period. (Figure 2).

Following four monthly administrations of Risperidone ISM $100 \mathrm{mg}$, the mean steady-state concentration versus time profiles for risperidone active moiety was characterized.

The median $\mathrm{T}_{\max }$ ss value was 48 hours, which may be skewed due to a presence of an expected secondary peak between approximately 18-22 days post-dose (Figure 2).

Statistical analysis of time to steady-state for the risperidone active moiety following repeated once monthly Risperidone ISM dosing and observation of the mean plasma concentration versus day profiles suggest that steady-state concentrations were achieved following Dose 1 and were maintained through Dose 4 (Supplementary Table 1).

Mean (SD) steady-state plasma concentrations for risperidone active moiety attained after the 4th monthly dose of Risperidone ISM were within the steady-state 
Table I Demographic and Baseline Characteristics (Safety Population)

\begin{tabular}{|c|c|}
\hline Variable & Value $\mathbf{N}=\mathbf{8}$ I \\
\hline \multicolumn{2}{|l|}{ Age (years) } \\
\hline Mean (SD) & $49.2(11.03)$ \\
\hline Min/Max & $20 / 65$ \\
\hline \multicolumn{2}{|l|}{ Sex, n (\%) } \\
\hline Female & $23(28.4)$ \\
\hline Male & $58(71.6)$ \\
\hline \multicolumn{2}{|l|}{ Race, n (\%) } \\
\hline White & $17(21.0)$ \\
\hline Black or African American & $62(76.5)$ \\
\hline Asian & $\mathrm{I}(\mathrm{I} .2)$ \\
\hline Other & $\mathrm{I}(\mathrm{I} .2)$ \\
\hline \multicolumn{2}{|l|}{ Ethnicity, n (\%) } \\
\hline Hispanic or Latino & $9(11.1)$ \\
\hline Not Hispanic or Latino & $72(88.9)$ \\
\hline \multicolumn{2}{|l|}{ Height $(\mathrm{cm})$} \\
\hline Mean (SD) & $172.17(7.3)$ \\
\hline $\operatorname{Min} / \operatorname{Max}$ & $152.5 / 185.3$ \\
\hline \multicolumn{2}{|l|}{ Weight (Kg) } \\
\hline Mean (SD) & $83.0(15.0)$ \\
\hline $\operatorname{Min} / \operatorname{Max}$ & $48.2 / 117.9$ \\
\hline \multicolumn{2}{|l|}{ BMI $\left(\mathrm{kg} / \mathrm{m}^{2}\right)$} \\
\hline Mean (SD) & $27.96(4.5)$ \\
\hline $\operatorname{Min} / \operatorname{Max}$ & $17.8 / 35.0$ \\
\hline
\end{tabular}

Abbreviation: SD, standard deviation.

$\mathrm{C}_{\min \mathrm{SS}}-\mathrm{C}_{\max } \mathrm{ss}$ range observed for the oral risperidone in this study (Figure 3).

Intersubject variability of steady-state (post-dose Day 7 [oral risperidone] and Day 92 [Risperidone ISM]) concentrations versus time profiles for risperidone active moiety presented a broader variability range for oral risperidone versus risperidone ISM, being the $\% \mathrm{CV}$ range $40-65 \%$ and $38 \%-52 \%$, respectively.

\section{Pharmacokinetic Parameters}

As shown in Table 2, following repeat administration of risperidone, mean steady-state peak $\left(\mathrm{C}_{\max } \mathrm{ss}\right)$, minimum $\left(\mathrm{C}_{\text {min ss }}\right)$, average $\left(\mathrm{C}_{\text {ave }}\right)$ and total $\left(\mathrm{AUC}_{\text {tau }}\right)$ (comparing ISM AUC $\mathrm{AUu}_{\text {tau }}$ to oral Adj. $\mathrm{AUC}_{\text {tau }}$ ) plasma exposure values for the risperidone active moiety were similar-to-slightly higher following $100 \mathrm{mg}$ Risperidone ISM compared to once daily $4 \mathrm{mg}$ oral risperidone. Fluctuation in risperidone active moiety concentrations over the profile was similar for risperidone ISM and oral risperidone, as Fluc values were $111 \%$ and $109 \%$ for oral risperidone and Risperidone ISM, respectively. The intersubject variability $(\% \mathrm{CV})$ for exposure parameters $\left(\mathrm{C}_{\max s \mathrm{ss}}, \mathrm{C}_{\mathrm{min} s \mathrm{ss}}, \mathrm{C}_{\mathrm{ave}}\right.$, and $\mathrm{AUC}_{\text {tau }}$ ) was moderate and similar between both treatments, with values ranging from $34-47 \%$ (Table 2).

\section{Comparative Bioavailability at Steady-State}

Following repeated administration of risperidone, minimum exposure $\left(\mathrm{C}_{\min s \mathrm{~s}}\right)$ to risperidone active moiety met bioequivalence criteria between treatments, with a geometric least square (LS) mean ratio (GMR) (risperidone ISM/oral risperidone) of 1.09 and a $90 \% \mathrm{CI}$ that was contained within the bioequivalence range of $0.80-1.25$. Additionally, the Fluc values also met bioequivalence criteria between the 2 treatments, as the GMR was 0.96 with a $90 \%$ CI that was contained within the bioequivalence range or $0.80-1.25$. Steady-state peak, total and average exposures to risperidone active moiety were slightly elevated for risperidone ISM compared to oral risperidone, with GMR $(90 \% \mathrm{CI})$ for $\mathrm{C}_{\max s \mathrm{ss}}, \mathrm{AUC}_{\mathrm{tau}}$, and $\mathrm{C}_{\text {ave }}$ of 1.17 (1.08-1.27), $1.24(1.16-1.33)$, and 1.24 (1.16-1.33), respectively; the upper $90 \%$ confidence bound was slightly outside the $0.80-1.25$ interval (Table 3).

Statistical analysis of time to steady-state for risperidone active moiety following repeated oral risperidone once daily dosing and Risperidone ISM once every 4 weeks using the Helmert Contrast Transformation showed that the geometric mean ratio (GMR) for each dose comparison fluctuated between $0.89-1.00$, and the $90 \%$ CIs of the GMRs contained 1 (Supplementary Table 1). The results of this analysis showed that steady-state concentrations were achieved for Risperidone ISM following Dose 1 and were maintained through Dose 4.

\section{Safety}

A total of 46 subjects (56.8\%) experienced at least one treatment-related TEAE, 26 (32.1\%) following oral risperidone and 34 (46.6\%) following Risperidone ISM (Supplementary Table 2). Sixteen (21.9\%) subjects reported at least one treatment-related TEAE after the first dose of Risperidone ISM, the most frequent being somnolence (11 subjects [15.1\%]) and increased appetite (2 subjects [2.7\%]) (Table 4). Two subjects (2.5\%) experienced treatment-related TEAEs that led to discontinuation: one subject $(1.2 \%)$ receiving oral risperidone 


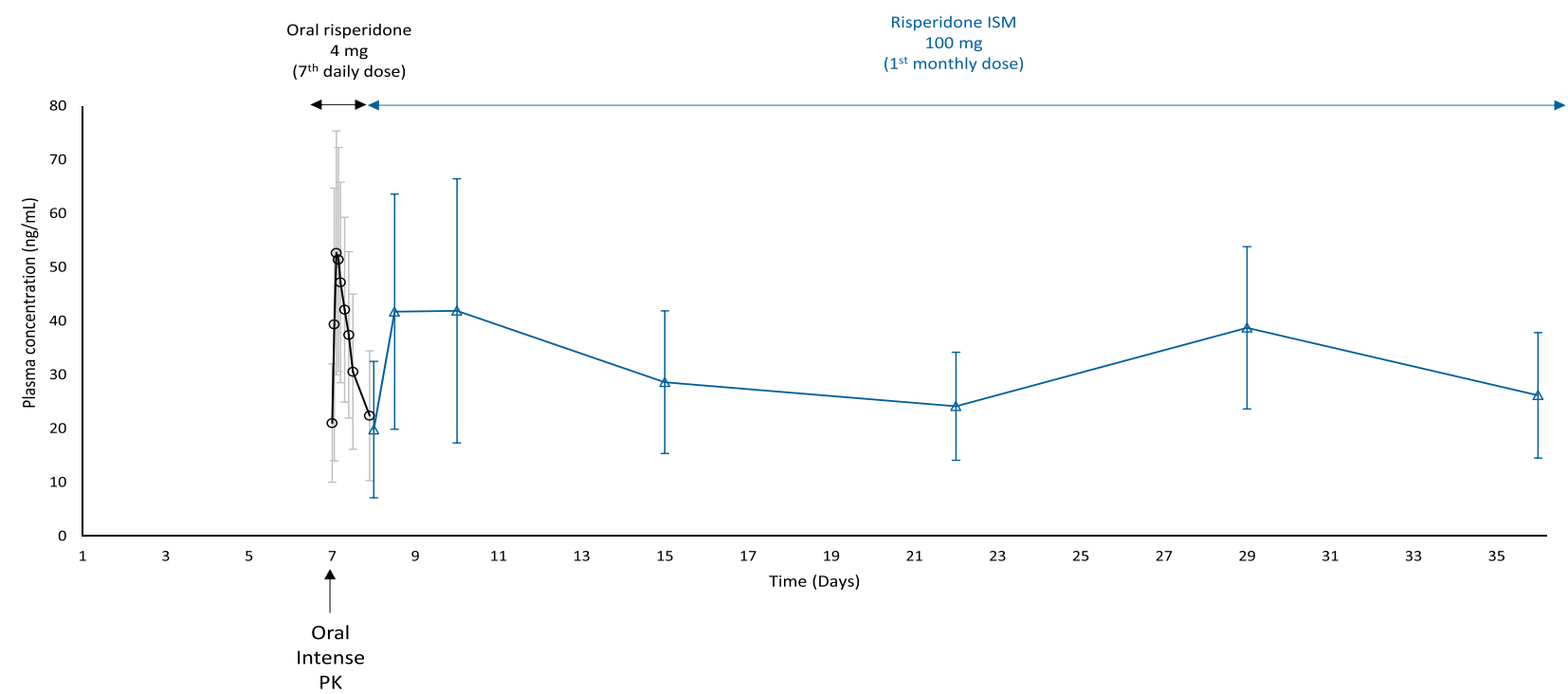

Figure 2 Mean $( \pm S D)$ plasma concentrations versus time profiles for risperidone active moiety during oral risperidone 4 mg treatment (7th dose) and after switching to risperidone ISM $100 \mathrm{mg}$ (PK population).

Notes: Once daily oral risperidone $4 \mathrm{mg}$ was administered for 7 days. An intense oral pharmacokinetic (PK) analysis was conducted at study Day 7 (last day of the oral risperidone treatment) including samples at pre-dose (within 0.5 hours relative to the dose time), I, 2, 3, 4, 6, 8, and I 2 hours, post-dose (black line). Twenty-four hours after the last oral risperidone dose (study Day 8 ), a single intramuscular dose of Risperidone ISM 100 mg was administered and PK samples were obtained at pre-dose and I 2 hours post-dose, as well as at Days 10, 15, 22, 29, and 36 (blue line).

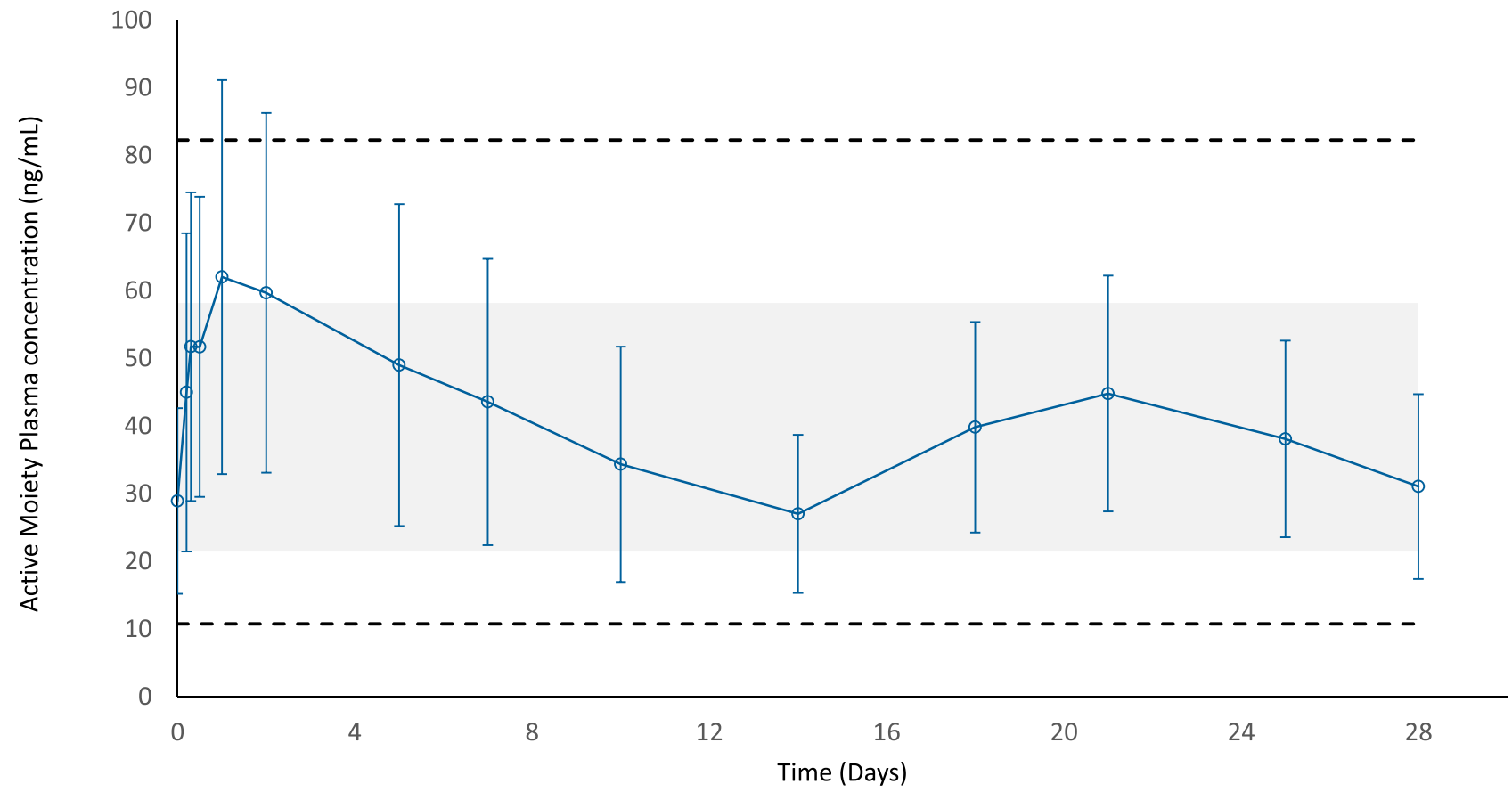

Figure 3 Mean $( \pm S D)$ steady-state plasma concentrations versus time profiles for risperidone active moiety after the 4 th monthly dose of Risperidone ISM I00 mg (PK population).

Notes: The blue line corresponds to the mean (SD) active moiety plasma concentrations of Risperidone ISM $100 \mathrm{mg}$. The shaded gray area corresponds to the $\mathrm{C}_{\mathrm{min}} \mathrm{ss}$ $\mathrm{C}_{\max s s}$ range observed after the 7 th once daily dose of oral risperidone $4 \mathrm{mg}$ (steady-state). Dashed black lines represent those $\mathrm{C}_{\min }$ minus $\mathrm{SD}$ at the bottom and $\mathrm{C}_{\max }$ plus $\mathrm{SD}$ at the top, for steady-state oral risperidone.

Abbreviations: $C_{\min s s}$, minimum plasma concentration at steady-state; $A U C_{\text {tau }}$, area under the curve during the dosing interval; $C_{\text {max ss }}$, maximum plasma concentration at steady-state. 
Table 2 Geometric Means (\%CV) for Steady-State Plasma Risperidone Active Moiety PK Parameters by Treatment (PK Population)

\begin{tabular}{|c|c|c|}
\hline PK Parameter & Oral Risperidone $\mathbf{N}=48$ & Risperidone ISM N=48 \\
\hline \multicolumn{3}{|l|}{ Tmax ss (h) } \\
\hline Median & 2.0 & 47.9 \\
\hline Minimum, maximum & $0.95,12.0$ & $2.0,670.4$ \\
\hline \multicolumn{3}{|l|}{$\mathrm{C}_{\max s \mathrm{~s}}(\mathrm{ng} / \mathrm{mL})$} \\
\hline Mean & 54.08 & 64.85 \\
\hline$\% C V$ & 39.7 & 39.8 \\
\hline \multicolumn{3}{|l|}{$\mathrm{C}_{\min \mathrm{ss}}(\mathrm{ng} / \mathrm{mL})$} \\
\hline Mean & 19.39 & 21.22 \\
\hline$\% C V$ & 46.6 & 41.3 \\
\hline \multicolumn{3}{|l|}{$\mathrm{C}_{\text {ave }}(\mathrm{ng} / \mathrm{nL})$} \\
\hline Mean & 30.52 & 38.63 \\
\hline$\% C V$ & 41.3 & 34.2 \\
\hline \multicolumn{3}{|l|}{ Fluc (\%) } \\
\hline Mean & 110.848 & 108.674 \\
\hline$\% \mathrm{CV}$ & 30.6 & 33.8 \\
\hline \multicolumn{3}{|c|}{$\mathrm{AUC}_{\mathrm{tau}}(\mathrm{h} * \mathrm{ng} / \mathrm{mL}[\mathrm{A}]$, day*ng/mL $[\mathrm{B}]$} \\
\hline Mean & 732.4 & 1082 \\
\hline$\% \mathrm{CV}$ & 41.3 & 34.2 \\
\hline \multicolumn{3}{|l|}{ Adj. AUC tau $($ day*ng/mL) } \\
\hline Mean & 854.5 & \\
\hline$\% C V$ & 41.3 & \\
\hline
\end{tabular}

Notes: $\mathrm{Adj} . \mathrm{AUC}_{\mathrm{tau}}=\mathrm{AUC}_{\mathrm{tau}} * 28$ (presented for oral risperidone treatment only) $\left(\mathrm{AUC}_{\mathrm{tau}}\right.$ was converted to $\mathrm{ng} *$ day $/ \mathrm{mL}$ before multiplying by 28$)$; $\mathrm{C}_{\mathrm{ave}}=\mathrm{AUC} \mathrm{C}_{\mathrm{tau} / \mathrm{tau}}(\mathrm{tau}=$ 24 hours and 28 days for oral and Risperidone ISM treatments, respectively); Fluc $=100 *\left(C_{\max s s}-C_{\min s s}\right) / C_{\text {ave }}$. PK parameters for oral risperidone treatment were estimated after the $7^{\text {th }}$ oral dose of risperidone. PK parameters for Risperidone ISM treatment were estimated after the 4th dose of Risperidone ISM. [A], Oral risperidone treatment= a single oral dose of $4 \mathrm{mg}$ risperidone once daily from Days I to 7; [B], Risperidone ISM treatment= once monthly (every 4 weeks) intramuscular dose of I00 mg risperidone ISM from Days 8 to 92.

Abbreviations: \%CV, coefficient of variation; $T_{\max s s}$, time to the maximum plasma concentration at steady-state; $h$, hours; $\mathrm{C}_{\max }$ ss, maximum plasma concentration at steady-state; $\mathrm{C}_{\min s \mathrm{~s}}$, minimum plasma concentration at steady-state; $\mathrm{C}_{\text {ave }}$, average plasma concentration; Fluc, percentage peak to trough fluctuation over a dosing interval; $\mathrm{AUC}_{\mathrm{tau}}$, area under the plasma concentration versus time curve during the dosing interval.

experienced sedation and one subject (1.4\%) receiving Risperidone ISM experienced akathisia. Globally, 84 treatment-related TEAEs were reported, most of them being $(98.8 \%)$ mild or moderate in intensity. The most common being somnolence (19.8\%), weight increase and hyperprolactinemia (18.5\% each) (Supplementary Table 2). No deaths were reported during the study. Two subjects, after receiving Risperidone ISM, experienced a total of 4 serious TEAEs (blood bilirubin increased, hyponatremia, hypokalemia and incarcerated inguinal hernia), and none of them were related to study drug.

Except for glucose, LDL cholesterol, and triglycerides, for which the mean values were slightly above the reference range in some time points assessed in the study, mean clinical chemistry, hematology and urinalysis results were within the reference ranges, and the mean values and changes from baseline were similar at each time point assessed after dosing for each treatment.

Mean prolactin values were above the reference range (reference range: 2.64 to $13.13 \mu \mathrm{g} / \mathrm{L}$ ) at some time points after receiving risperidone (oral and ISM), but only mild changes from baseline in mean prolactin values were observed after both treatments and did not lead to treatment discontinuation.

In three subjects a mild swelling at injection site was recorded; none of these injection site reactions were considered clinically significant.

There were no clinically significant changes in 12-lead ECG measurements, nor in QT intervals. No notable changes were observed on C-SSRS, SAS, BARS, AIMS scales.

Regarding the CGI-S scale, mean scores were 3.3 at all visits for both treatments, from baseline throughout the remainder of the study visits. 
Table 3 Statistical Analysis of Comparative Bioavailability for Risperidone Active Moiety PK Parameters at Steady-State (PK Population)

\begin{tabular}{|c|c|c|c|c|c|}
\hline \multirow[t]{2}{*}{ PK Parameter } & \multirow[t]{2}{*}{ Treatment } & \multirow{2}{*}{$\begin{array}{c}\text { Geometric LS } \\
\text { Means }\end{array}$} & \multirow{2}{*}{$\begin{array}{c}\text { Geometric LS } \\
\text { Means }\end{array}$} & \multicolumn{2}{|c|}{$90 \% \mathrm{Cl}$ of the Ratio } \\
\hline & & & & Lower & Upper \\
\hline Adj. $A \cup C_{\text {tau }}($ day*ng/mL) & $\begin{array}{l}\text { Oral } \\
\text { ISM }\end{array}$ & $\begin{array}{r}854.5 \\
1063\end{array}$ & 1.2439 & 1.1600 & 1.3339 \\
\hline$C_{\max s s}(n g / m L)$ & $\begin{array}{l}\text { Oral } \\
\text { ISM }\end{array}$ & $\begin{array}{l}54.08 \\
63.08\end{array}$ & I.1664 & 1.0750 & 1.2655 \\
\hline$C_{\min s s}(\mathrm{ng} / \mathrm{mL})$ & $\begin{array}{l}\text { Oral } \\
\text { ISM }\end{array}$ & $\begin{array}{l}19.39 \\
21.08\end{array}$ & 1.0869 & 0.9930 & 1.1896 \\
\hline $\mathrm{C}_{\text {ave }}(\mathrm{ng} / \mathrm{nL})$ & $\begin{array}{l}\text { Oral } \\
\text { ISM }\end{array}$ & $\begin{array}{l}30.52 \\
37.96\end{array}$ & 1.2439 & 1.1600 & 1.3339 \\
\hline Fluc (\%) & $\begin{array}{l}\text { Oral } \\
\text { ISM }\end{array}$ & $\begin{array}{l}110.848 \\
106.949\end{array}$ & 0.9648 & 0.8714 & 1.0683 \\
\hline
\end{tabular}

Notes: N=48; treatment comparison: once monthly (every 4 weeks) intramuscular dose of Risperidone ISM $100 \mathrm{mg}$ (from Days 8 to 92) versus once daily oral dose of risperidone $4 \mathrm{mg}$ (from Days I to 7). $\mathrm{Adj}$. $A \cup \mathrm{C}_{\text {tau }}=\mathrm{AUC} \mathrm{C}_{\mathrm{tau}}{ }^{*} 28$ (presented for risperidone oral treatment only) $\left(\mathrm{AUC}_{\mathrm{tau}}\right.$ was converted to ng*day/mL before multiplying by 28). Adj. $\mathrm{AUC}_{\mathrm{tau}}$ used for oral risperidone treatment. An ANOVA with treatment as a fixed effect was fitted to each log-transformed PK parameter. Results were backtransformed to obtain the geometric LS mean, geometric LS mean ratio, and the $90 \% \mathrm{Cl}$. PK parameters for oral risperidone treatment were estimated after the 7th oral dose of risperidone. PK parameters for Risperidone ISM treatment were estimated after the 4th dose of Risperidone ISM.

Abbreviations: $C_{\max \text { ss }}$, maximum plasma concentration at steady-state; $C_{\min s s}$, minimum plasma concentration at steady-state; $C_{\text {ave }}$, average plasma concentration; Fluc, percentage peak to trough fluctuation over a dosing interval; $A \cup C_{\text {tau, }}$, area under the plasma concentration versus time curve during the dosing interval.

Table 4 Summary of Treatment-Related TEAEs by Each Monthly Dose of Risperidone ISM 100 mg (Safety Population)

\begin{tabular}{|c|c|c|c|c|}
\hline \multirow[t]{2}{*}{ Preferred Term } & I st Dose $\mathbf{N}=\mathbf{8}$ | & 2nd Dose $N=67$ & 3rd Dose $N=61$ & 4th Dose $N=58$ \\
\hline & n (\%) & n (\%) & n (\%) & n (\%) \\
\hline Subjects with treatment-related TEAEs & $16(21.9)$ & $7(10.4)$ & $4(6.6)$ & $15(25.9)$ \\
\hline Akathisia & 0 & $3(4.5)$ & 0 & $\mathrm{I}(\mathrm{I} .7)$ \\
\hline Constipation & $\mathrm{I}(\mathrm{I} .4)$ & 0 & 0 & 0 \\
\hline Headache & 0 & $\mathrm{I}(\mathrm{I} .5)$ & 0 & 0 \\
\hline Hyperprolactinemia & $\mathrm{I}(\mathrm{I} .4)$ & $\mathrm{I}(\mathrm{l} .5)$ & $3(4.9)$ & 0 \\
\hline Increased appetite & $2(2.7)$ & 0 & $\mathrm{I}(\mathrm{I} .6)$ & 0 \\
\hline Somnolence & II (I5.I) & 0 & 0 & 0 \\
\hline Weight increased & 0 & $\mathrm{I}(\mathrm{I} .5)$ & I ( 1.6$)$ & $13(22.4)$ \\
\hline
\end{tabular}

Notes: Descriptions of TEAEs are coded using the Medical Dictionary for Regulatory Activities (MedDRA), version 21. Treatment-related TEAEs listed occurred in $\geq 2 \%$ of patients.

Abbreviation: TEAEs, treatment-emergent adverse events.

\section{Discussion}

The aim of this study was to evaluate the steady-state comparative bioavailability of Risperidone ISM with oral risperidone. Data obtained here demonstrate that once monthly IM injections of Risperidone ISM $100 \mathrm{mg}$ were an appropriate treatment for stable subjects treated with $4 \mathrm{mg}$ /day or higher oral risperidone. These results demonstrate that there is no time lag in achieving plasma concentration comparable with the oral formulation after the first dose of Risperidone ISM. This finding demonstrates that the direct switch from oral risperidone to Risperidone ISM can be made 24 hours after the last oral dose, since steady-state concentrations are evidently maintained within the same range of concentration for active moiety obtained with the oral formulation without the need for loading doses or oral risperidone supplementation. Other monthly LAI atypical antipsychotics, such as paliperidone palmitate (PP) or aripiprazole, require a loading dose to attain steady-state concentrations when switching from oral treatment to their injectable formulations because they do not have a rapid optimal release. $^{15,16}$ In fact, in the 7-day period following the first IM injection of PP, the median plasma 
concentrations of paliperidone gradually decreased to close to the minimum therapeutic level of $7.5 \mathrm{ng} / \mathrm{mL}$ in the median plasma concentrations of PP $(7.59 \mathrm{ng} / \mathrm{mL}$ for the PP $50 \mathrm{mg}$ eq and $8.24 \mathrm{ng} / \mathrm{mL}$ for the PP $100 \mathrm{mg}$ eq), as Kramer et al published. ${ }^{17}$ Besides, with the two ways to initiate treatment with aripiprazole monthly or aripiprazole lauroxil, not only a loading dose, but an oral aripiprazole supplementation is also needed to maintain therapeutic concentrations during initiation of therapy. ${ }^{16,18}$

The steady-state risperidone active moiety $\mathrm{PK}$ parameters $\left(\mathrm{C}_{\max }\right.$ ss, $\mathrm{C}_{\min }$ ss, $\mathrm{C}_{\mathrm{ave}}$ and $\left.\mathrm{AUC}_{\text {tau }}\right)$ for monthly injections of $100 \mathrm{mg}$ Risperidone ISM were similar or slightly higher than daily doses of $4 \mathrm{mg}$ oral risperidone. Fluctuation in risperidone active moiety concentrations over the profile was also similar for both treatments. Specifically, $\mathrm{C}_{\text {min ss }}$ plasma exposure to risperidone active moiety and fluctuation in plasma concentrations (Fluc) of risperidone active moiety met bioequivalence criteria between treatments, while $\mathrm{C}_{\max \text { ss }}, \mathrm{AUC}_{\text {tau }}$, and $\mathrm{C}_{\text {ave }}$ were only slightly higher for Risperidone ISM compared to oral risperidone, the upper $90 \%$ confidence bound being marginally outside the $0.80-1.25$ interval for all three measures. These results substantiate a sustained release of risperidone from the Risperidone ISM long-acting injectable formulation.

Intersubject variability for the steady-state concentrations versus time profiles for risperidone active moiety presented a broader variability range for oral risperidone compared with Risperidone ISM. As reported previously, when oral and long-acting IM formulations of typical antipsychotics have been compared at steady-state, the variability in the range of plasma concentrations at a given IM dose has been lower than with oral dosing. ${ }^{19}$ This appears to be related to a more controlled and constant release combined with the circumvention of first-pass metabolism with long-acting IM formulations. ${ }^{20}$

Both risperidone treatments (oral and Risperidone ISM) were well tolerated. It should be noted that direct comparisons on safety data between both study treatments should be interpreted with caution as the duration of each treatment period was different ( 7 days with oral risperidone and 16 weeks with Risperidone ISM). Nevertheless, overall, no new safety signals were detected, and the adverse events observed were those expected for risperidone at therapeutic doses. $^{21,22}$ Furthermore, the TEAEs reported were in line with those observed in previous studies with Risperidone $\mathrm{ISM}^{4,5}$ and the overall dropout rate was also in agreement with those reported in other studies with antipsychotics. ${ }^{23-25}$
Most treatment-related TEAEs reported were mild or moderate in severity, leading to study drug discontinuation in only two subjects $(2.5 \%)$, one due to sedation whilst receiving oral treatment and one due to akathisia following a Risperidone ISM dose.

Increased prolactin levels were one of the more frequently reported TEAEs in both treatments although none of them led to study discontinuation and the incidence was consistent with that observed in other studies. ${ }^{26,27}$ Nevertheless, interestingly, the incidence of treatment-related hyperprolactinemia decreased to $6.8 \%$ after treatment with Risperidone ISM compared to $12.3 \%$ during the oral period.

Safety and tolerability data, along with the PK findings, provide further assurances that switching from oral risperidone to Risperidone ISM IM injection treatment is well tolerated and adequate to maintain steady-state active moiety levels throughout the first month and beyond.

Several limitations need to be considered when interpreting the study results. The open-label nature of this study was a potential source of bias, as well as the limited number of patients included or that two cross-over arms were not foreseen, but we do not believe that these limitations detract from the conclusions drawn because the sample size and study design were appropriate to achieve the objectives set in the study, and although it was not designed to evaluate efficacy, no changes were shown in the CGI-S score, confirming the stability of subjects during treatment with Risperidone ISM.

\section{Conclusion}

In conclusion, this study provides evidence that steady-state minimum plasma exposure and fluctuation in plasma concentrations of risperidone active moiety were similar. Moreover, steady-state total and peak plasma exposures of risperidone active moiety were only slightly higher following monthly IM Risperidone ISM $100 \mathrm{mg}$ compared to once daily oral risperidone $4 \mathrm{mg}$. Both risperidone treatments, oral and Risperidone ISM were well tolerated. The ability to rapidly transition from oral to injectable is due to the rapid release of Risperidone ISM, which allows achieving the desired therapeutic plasma levels similar to those observed at the steady-state after oral risperidone treatment. Therefore, direct switch after 24 hours from the last oral risperidone dose to Risperidone ISM treatment may be done in schizophrenia patients with no time lag, maintaining steady-state levels of the active moiety throughout the treatment without the need for oral supplementation or loading doses. 


\section{Principal Investigators and Sites}

David P Walling, Collaborative Neuroscience Network, Garden Grove, California, USA. Howard A. Hassman, Hassman Research Institute, Berlin, New Jersey, USA.

\section{Data Sharing Statement}

Risperidone ISM is currently under evaluation by the Health Authorities. Therefore, any data beside what are included in the manuscript will not be available until the process resolution. Data supporting the results of this study are available from ROVI, but restrictions apply to the availability of these data, which were used under license for the present study and are therefore not publicly available. However, the data may be provided by the corresponding author upon reasonable request and with the permission of ROVI.

\section{Ethics Approval and Informed Consent}

The Ethics approval and the informed consent form were approved by Ethics Committee for each site:

For Collaborative Neuroscience Network:

Alpha Independent Review Board (IRB)

1001 Avenida Pico, Suite C \# 497, San Clemente, CA 92673

For Hassman Research Institute:

Aspire Independent Review Board (IRB)

11491 Woodside Avenue

Santee, CA 92071.

\section{Acknowledgments}

The authors would like to acknowledge Begoña Gorostidi, Jordi Llaudó, Ana Belén López-Yélamos and Mateo Fernández-Varela, for their contributions to the study. The authors thank Carl Sherifi and Fernando Zaragozá for their language review of the manuscript. All of them are employees of Laboratorios Farmacéuticos ROVI, S.A. The authors also thank the study participants, their families and caregivers, and the investigators for their participation.

\section{Author Contributions}

All authors made substantial contributions to conception and design, acquisition of data, or analysis and interpretation of data; took part in drafting the article or revising it critically for important intellectual content; agreed to submit to the current journal; gave final approval of the version to be published; and agree to be accountable for all aspects of the work.

\section{Funding}

This study was funded by Laboratorios Farmacéuticos Rovi, S.A. Madrid, Spain. It was supported also in part by Centro para el Desarrollo Tecnológico Industrial IDI20180128.

\section{Disclosure}

Lourdes Anta, Lourdes Ochoa, Ignacio Ayani, Javier Martínez and Ibon Gutierro are employees of Laboratorios Farmacéuticos Rovi, S.A., the sole developer of Risperidone ISM. Dr Javier Martínez also reports grants from Centro para el Desarrollo Tecnológico Industrial, during the conduct of the study. David P Walling has received grant funding from Novartis, Janssen, Intracellular, Lyndra, Rovi, Otsuka, Alkermes, Cerevel, AbbVie, Allergan, Noven, Takeda, Indivior, Avanir, Lundbeck, Roche, Boehringer Ingelheim, Biogen, Sunovion and Acadia. He has served on Advisory Boards for Otsuka, Janssen, Biogen, Boehringer Ingelheim and Lyndra. Howard A. Hassman declared that he has no competing interests. The authors report no other conflicts of interest in this work.

\section{References}

1. Ascher-Svanum H, Faries DE, Zhu B, Ernst FR, Swartz MS, Swanson JW. Medication adherence and long-term functional outcomes in the treatment of schizophrenia in usual care. $J$ Clin Psychiatry. 2006;67(3):453-460. doi:10.4088/JCP.v67n0317

2. Correll CU, Citrome L, Haddad PM, et al. The use of long-acting injectable antipsychotics in schizophrenia: evaluating the evidence. J Clin Psychiatry. 2016;77(Suppl 3):1-24. doi:10.4088/JCP.15032su1

3. Correll CU, Kim E, Sliwa JK, et al. Pharmacokinetic characteristics of long-acting injectable antipsychotics for schizophrenia: an overview. CNS Drugs. 2021;35:39-59. doi:10.1007/s40263-020-00779-5

4. Llaudó J, Anta L, Ayani I, et al. Open-label, randomized, parallel study to evaluate the pharmacokinetics, safety, and tolerability of one intramuscular injection of risperidone ISM at different dose strengths in patients with schizophrenia or schizoaffective disorder (PRISMA-1). Int Clin Psychopharmacol. 2016;31:323-331. doi:10.1097/YIC.0000000000000139

5. Anta L, Llaudó J, Ayani I, Martínez J, Litman RE, Gutierro I. A Phase II study to evaluate the pharmacokinetics, safety, and tolerability of risperidone ISM multiple intramuscular injections once every 4 weeks in patients with schizophrenia. Int Clin Psychopharmacol. 2018;33:9-87.

6. Anta L, Mata E, Ochoa Díaz de Monasterioguren L. Newer Formulations of risperidone: remarks about risperidone ISM. CNS Drugs. 2020;34(10):1087-1088. doi:10.1007/s40263-020-00762-0

7. Guy W. The Clinician Global Severity and Impression Scales. ECDEU Assessment Manual for Psychopharmacology. Superintendent of Documents, IS Government Printing Office, Publication No. 76-338. Washington, DC: US Department of Health, Education, and Welfare; 1976a:218-222.

8. Posner K, Oquendo MA, Gould M, Stanley B, Davies M. Columbia Classification Algorithm of Suicide Assessment (C-CASA): classification of suicidal events in the FDA's pediatric suicidal risk analysis of antidepressants. Am J Psychiatry. 2007;164:1035-1043. doi:10.1176/ ajp.2007.164.7.1035 
9. Guy W. Abnormal Involuntary Movement Scale (AIMS). ECDEU Assessment Manual for Psychopharmacology. Rockville, MD: US Dept. of Health, Education and Welfare, Public Health Service, Alcohol, Drug Abuse, and Mental Health Administration, National Institute of Mental Health, Psychopharmacology Research Branch, Division of Extramural Research Programs; 1976b:534-537.

10. Barnes TR. A rating scale for drug-induced akathisia. $\mathrm{Br}$ J Psychiatry. 1989;154:672-676. doi:10.1192/bjp.154.5.672

11. Simpson GM, Angus JW. A rating scale for extrapyramidal side effects. Acta Psychiatr Scand. 1970;1970(Suppl 212):11-19. doi:10.1111/j.1600-0447.1970.tb02066.x

12. Maganti L, Panebianco DL, Maes AL. Evaluation of methods for estimating time to steady-state with examples from Phase 1 studies. AAPS J. 2008;10:141-147. doi:10.1208/s12248-008-9014-y

13. Newlands A. Statistics and pharmacokinetics in clinical pharmacology studies (paper ST03). Presented at: SAS Conference Proceedings: pharmaceutical Users Software Exchange; PhUSE Dublin; 9-11 October; 2006; Dublin, Ireland.

14. Samtani M, Gopal S, Gassmann-Mayer C, Alphs L, Palumbo JM. Dosing and switching strategies for paliperidone palmitate: based on population pharmacokinetic modelling and clinical trial data. CNS Drugs. 2011;25:829-845.

15. Xeplion ${ }^{\circledR}$ summary of product characteristics. Available from: https:// www.ema.europa.eu/en/documents/product-information/xeplion-epar -product-information_en.pdf. Accessed July 20, 2021.

16. Abilify maintena ${ }^{\circledR}$ summary of product characteristics. Available from: https://www.ema.europa.eu/en/documents/product-information /abilify-maintena-epar-product-information_en.pdf. Accessed July 20, 2021.

17. Kramer M, Litman R, Hough D, et al. Paliperidone palmitate, a potential long-acting treatment for patients with schizophrenia. Results of a randomized, double-blind, placebo-controlled efficacy and safety study. Int J Neuropsychopharmacol. 2010;13(5):635-647. doi:10.1017/S1461145709990988

18. Aristada Full Prescribing Information. Available from: https://www. aristadahcp.com/downloadables/ARISTADA-INITIO-PI.pdf. Accessed July 26, 2021.
19. Tavcar R, Dernovsek MZ, Zvan V. Choosing antipsychotic maintenance therapy-a naturalistic study. Pharmacopsychiatry. 2000;33 (2):66-71. doi:10.1055/s-2000-7969

20. Ereshefsky L, Saklad SR, Jann MW, Davis CM, Richards A, Seidel DR. Future of depot neuroleptic therapy: pharmacokinetic and pharmacodynamic approaches. $J$ Clin Psychiatry. 1984;45:50-59.

21. Risperdal ${ }^{\circledR}$ (oral risperidone) [prescribing information]. Available from: https:/www.accessdata.fda.gov/drugsatfda_docs/label/2020/ 020272s085,020588s072,021444s0581bl.pdf. Accessed July 1, 2021.

22. Song F. Risperidone in the treatment of schizophrenia: a meta-analysis of randomized controlled trials. J Psychopharmacol. 1997;11(1):65-71. doi:10.1177/026988119701100116

23. Mamo D, Kapur S, Keshavan M, et al. D 2 receptor occupancy of olanzapine pamoate depot using positron emission tomography: an open-label study in patients with schizophrenia. Neuropsychopharmacology. 2008;33(2):298-304. doi:10.1038/sj. npp.1301409

24. Mamo D, Kapur S, Shammi CM, et al. A PET study of dopamine D2 and serotonin 5-HT2 receptor occupancy in patients with schizophrenia treated with therapeutic doses of ziprasidone. Am J Psychiatry. 2004;161(5):818-825. doi:10.1176/appi.ajp.161.5.818

25. Thyssen A, Rusch S, Herben V, Quiroz J, Mannaert E. Risperidone long-acting injection: pharmacokinetics following administration in deltoid versus gluteal muscle in schizophrenic patients. $J$ Clin Pharmacol. 2010;50(9):1011-1021. doi:10.1177/0091270009355156

26. Bushe C, Shaw M, Peveler RC. A review of the association between antipsychotic use and hyperprolactinaemia. J Psychopharmacol. 2008;22(Suppl):46-55. doi:10.1177/0269881107088435

27. Huhn M, Nikolakopoulou A, Schneider-Thoma J, et al. Comparative efficacy and tolerability of 32 oral antipsychotics for the acute treatment of adults with multi-episode schizophrenia: a systematic review and network meta-analysis. Lancet. 2019;394(10202):939-951. doi:10.1016/S0140-6736(19)31135-3

\section{Publish your work in this journal}

Drug Design, Development and Therapy is an international, peerreviewed open-access journal that spans the spectrum of drug design and development through to clinical applications. Clinical outcomes, patient safety, and programs for the development and effective, safe, and sustained use of medicines are a feature of the journal, which has also been accepted for indexing on PubMed Central. The manuscript management system is completely online and includes a very quick and fair peer-review system, which is all easy to use. Visit http://www. dovepress.com/testimonials.php to read real quotes from published authors. 